\title{
Multilinguales
}

Multilinguales

14 | 2020

Enseignement universitaire et professionnalisation : enjeux, questionnements et défis socio-économiques

\section{Penser l'écriture transitionnelle chez Claude Simon}

Thinking of transitional writing at Claude Simon

\section{Sinan Anzoumana}

\section{(2) OpenEdition \\ Journals}

Édition électronique

URL : https://journals.openedition.org/multilinguales/5884

DOI : $10.4000 /$ multilinguales.5884

ISSN : 2335-1853

Éditeur

Université Abderrahmane Mira - Bejaia

Référence électronique

Sinan Anzoumana, « Penser l'écriture transitionnelle chez Claude Simon », Multilinguales [En ligne], 14 | 2020, mis en ligne le 10 décembre 2020, consulté le 02 juillet 2021. URL : http://

journals.openedition.org/multilinguales/5884; DOI : https://doi.org/10.4000/multilinguales.5884

Ce document a été généré automatiquement le 2 juillet 2021.

\section{c) $($ ()) $\Theta$}

Multilinguales est mise à disposition selon les termes de la Licence Creative Commons Attribution -

Pas d'Utilisation Commerciale - Pas de Modification 4.0 International 


\title{
Penser l'écriture transitionnelle chez Claude Simon
}

\author{
Thinking of transitional writing at Claude Simon
}

\author{
Sinan Anzoumana
}

1 Alastair B. Duncan, dans l'introduction de Claude Simon : œuvres ${ }^{1}$ soulève un problème crucial, celui de la réception de l'œuvre de Claude Simon :

Pendant trop longtemps, l'univers des romans de Claude Simon est resté méconnu.

La réception n'a pas été à la hauteur de l'œuvre. Cela est dû en grande partie au

contexte dans lequel on lisait alors ses livres (Duncan, 2006 : XII).

Il est vrai que, comme l'écrit Alastair Duncan :

[...] l'association entre Claude Simon et le nouveau roman nuit à la réception de son œuvre. Le label lui reste trop longtemps attaché et masque ce qui est spécifique à son travail. [...] Cette critique théorisante a donc su éclairer des aspects de la forme des romans de Simon. Mais elle a mal servi l'écrivain en enfermant son travail dans un cadre trop rigide, [...]» (Duncan, 2006 : XIV)

Il s'agira au fond de croire que, libérées des excès de leur effet de posture, ces approches conduisent in fine chez Simon ce que nous appelons écriture transitionnelle ${ }^{2}$. Cette écriture traduit bien, chez lui, le lien entre le Nouveau roman et le postmodernisme littéraire qui avait été déjà annoncé.

3 Notre objectif consiste à interroger, à partir de quelques textes ${ }^{3}$, les manières dont cette écriture se dynamise pour pouvoir se libérer des conventions traditionnelles du roman. D'une manière significative, il s'agira de montrer comment cette écriture simonienne se légitime vis-à-vis des théories néo-romanesques d'une part et de l'autre, vis-à-vis des approches postmodernes, adoptant pour finir, une posture de l'entredeux.

Quelques questions fondamentales méritent dès lors d'être soulevées : d'un point de vue théorique, quels sont les éléments textuels et formels fondant ces rapprochements avec le Nouveau roman et le postmodernisme littéraire? Quel est l'enjeu qui sous-tend une telle aventure scripturale? Quelle esthétique ressortit à cette vision simonienne de l'écriture romanesque? 
Cette problématique d'ordre épistémologique nous conduira, dès l'abord, à l'appréciation de la portée néo-romanesque des textes de Simon en nous fondant notamment sur la ré(é)criture dûment élaborée par Anne-Claire Gignoux. Nous examinerons par la suite quelques traits significatifs des stratégies discursives et formelles du postmodernisme littéraire en jeu, en suivant les approches conceptuelles de Janet M. Paterson. Nous envisagerons in extremis comment les deux labels littéraires se neutralisent pour produire en fin de compte du nouveau sens, une nouvelle esthétique chez l'auteur :la ductilité et la transcendance de sa conception romanesque.

\section{In-scription : l'expression du nouveau roman chez Simon}

5 Claude Simon a reconnu que le contact avec les écrivains ${ }^{4}$ que Jérôme Lindon et Alain Robbe-Grillet ont rassemblé aux éditions de Minuit dans les années 1950 lui a été profitable dans son aventure scripturale :

«[...] Je considère comme une véritable chance de m'être trouvé au contact d'un groupe d'écrivains animés de préoccupations sinon identiques, du moins qui se recoupaient souvent, et ces échanges m'ont été d'une aide précieuse sans laquelle, très certainement, mon travail et ses résultats n'auraient pas été les mêmes (Simon, 1975 : 404).

Apparemment, c'est ce qui a poussé Katerine Gosselin à soutenir qu' :

$\mathrm{Au}$ tournant des années soixante, et jusqu'au début des années soixante-dix, le roman simonien et le Nouveau Roman ont pris leur place ensemble sur la scène littéraire, s'affirmant l'un avec l'autre, et trouvant l'un dans l'autre une part de leur légitimation (Gosselin,2011:69-85).

7 Pour autant, la manière dont Simon lui-même expose son rapport au Nouveau roman paraît ambiguë et marquée par le sceau de la disjonction. D'ailleurs, il est resté constant, logique et cohérent dans ses déclarations. En effet, le Prix Nobel de littérature 1985 n'a jamais manifesté ouvertement son appartenance à un mouvement littéraire, ni encore moins avoir été un fervent adepte de la théorisation du Nouveau roman. À ce sujet, il partagea, en 2007, une anecdote pleine d'humour avec la journaliste Monique Joguet :

Peu de temps avant sa mort, Merleau-Ponty avait consacré l'une de ses conférences au Collège de France à certains de mes romans, en particulier Le vent, L'herbe et La route des Flandres. Et je ne vous cache pas que ce qu'il avait vu dans ces romans, en particulier en ce qui concernait les dimensions temporelles et spatiales, me dépassait un peu, en tout cas j'étais un peu perdu dans le vocabulaire philosophique qui était le sien, $[. . .]^{5}$ (Simon, $2007: 169$ ).

Selon ses propres propos, il s'est senti «un peu perdu » avec ces «histoires » de théories. Car si la critique a retrouvé des traces d'une quelconque théorie dans ses romans, Claude Simon affirme qu' « [...] il n'y avait rien là chez moi de conscient ou intentionnel » (Dällenbach,1988: 168) d'autant plus que l'idée d'un livre lui venait justement autour d'un évènement ou d'un personnage (171).Cela amène Gosselin à reconnaître aussi qu'aucun programme ne prédéterminait les romans de Simon au Nouveau roman; elle mentionne dans le même texte que «Même dans les années où le Nouveau Roman s'imposait sur la scène littéraire [Claude Simon] a toujours mis en doute l'existence du Nouveau Roman » (Gosselin,2011: 69-85). En effet, à plus d'une reprise, Simon fut l'un des premiers écrivains avoir remis en cause l'existence même de 
cette mouvance littéraire et à signifier ouvertement les clivages qui existaient déjà entre eux. Pour preuve, en 1967, dans un entretien avec Madeleine Chapsal, il reconnait certes un rapprochement entre eux - les précurseurs du Nouveau roman -, mais il s'emploie davantage à relever les nuances, les divergences : «[...] nous nous trouvons spontanément d'accord pour rejeter un certain nombre de conventions qui régissent le roman traditionnel. Mais à partir de là, chacun de nous œuvre selon son tempérament» (Chapsal,1967: 4). Avec Alain Poirson et Jean-Paul Goux en 1977, l'écrivain ne varie pas dans sa position:

Nous avons tous eu en commun je crois - ou du moins j'ai cru le sentir - un certain nombre d'idées, plus ou moins formulées, plutôt négations d'ailleurs : des refus. [...] À partir de là, naturellement chacun de nous a suivi ses propres voies (heureusement ; sans quoi nous aurions tous écrit le même roman : ce qui aurait été plutôt ennuyeux...). Et dans des directions fort différentes. Sinon même opposées (Dällenbach,1988 : 159).

9 En 1985, avec Marianne Alphant, il reste fidèle à cette posture en déclarant : "Nous avons toujours en commun que nous répugnons au roman traditionnel, à ses formes sclérosées. À partir de là, chacun de nous a fait ce qu'il devait faire " (Alphant,1985 : 28). Cette orientation de Claude Simon est bien résumée par Roger-Michel Allemand pour qui «Les auteurs plus ou moins durablement associés à la mouvance néo-romanesque l'ont vécue de manière diverse. » (Allemand, 2013 : 144). En effet, bien des désaccords sont survenus entre Simon et ses congénères, en l'occurrence avec Ricardou dont il récusait le dogmatisme en 1985. Très tôt déjà, ses «amis » lui reprochaient, dans ses textes, l'usage de référents socioculturels pouvant engendrer un danger potentiel, celui d'un retour à « l'illusion référentielle » ainsi que le réalisme de ses descriptions. Mais Simon n'en démord pas ; il persévère dans sa voie avec, pour clé, la définition de Robbe-Grillet du «nouveau roman » :

appellation commode englobant tous ceux qui cherchent de nouvelles formes romanesques, capables d'exprimer (ou de créer) de nouvelles relations entre l'homme et le monde, tous ceux qui sont décidés à inventer le roman, c'est-à-dire, inventer l'homme (Robbe-Grillet,1961: 9)

Cette approche robbe-grilletienne est, rappelons-le, en parfaite contradiction avec la vision d'Émile Henriot ${ }^{6}$. Dans son discours de Stockholm, Claude Simon revenant sur son travail fera d'ailleurs part de sa nature artisanale. Dans ce travail d'artisan, l'auteur use de procédés de rupture notamment le fragment dont l'objectif serait la rénovation des formes du récit, débarrassées des carcans traditionnels.

10 Quoi qu'il en soit, ces quelques éléments paratextuels susmentionnés entretiennent des non-dits par rapport à un rapprochement avec le Nouveau roman. Bien plus, plusieurs éléments textuels et formels de l'œuvre simonienne ont été analysés à partir des théories néo-romanesques, trahissant ainsi les réflexions de l'auteur en attestant la proximité évoquée par la critique ${ }^{7}$. Nous ferons certes l'économie de telles analyses théoriques pertinentes et judicieuses, mais en reconsidérant ici, dans un développement non exhaustif, ce que la théorie qualifie de ré(é) criture $^{8}$. Pour ce faire, nous voulons pour preuve la recrudescence et la récurrence du « déjà vu », du " déjà lu » qui semblent charrier l'écriture simonienne dans la dimension néo-romanesque.

11 Anne-Claire Gignoux abordant la ré(é)criture comme l'un des traits consubstantiels du Nouveau roman formule que :

La récriture travaille le texte. Elle travaille le texte récrit: elle le découpe, le déforme, le détourne, ou le cite avec un respect obstiné. Elle se présente alors sous deux catégories très générales : une récriture exacte, qui cite littéralement, ou une 
récriture avec variation. Cependant, dans les eux cas, et même si la citation est totalement exacte, la récriture fait subir aux mots récrits un traitement, un travail toujours doublement transformateur : il modifie le texte récrit, fragmenté, exilé de son contexte, éventuellement corrigé, mais aussi le texte où il s'insère et qu'il enrichit. La récriture est polyphonique : mélange de voix, de mots nouveaux, mots répétés à reconnaître... (Gignoux,2003:7)

À partir de cette approche, il appert que les textes de l'écrivain tissent des liens inextricables avec d'autres textes antérieurs, contemporains qu'ils reprennent, imitent ou transforment à l'instar des textes religieux - surtout la Bible - sur lesquels nous voulons insister. Dès lors, pour parler comme Philippe Sellier, Simon « fait baigner le présent dans le sacré " (Sellier,1984: 114). Jean Duchesne cité par Paul Chautard renchérira en ces termes: «[...] il lui est tout simplement impossible d'empêcher que son œuvre ait une portée théologique, un enjeu spirituel » (Chautard,2012:12).

Dans cette perspective, examinons de près cet extrait d'Histoire :

Je suis Celui qui suis il vint vers eux pauvres pêcheurs tirant leurs filets vides pardonnez-nous pauvres pécheurs peinture de qui ? grisâtre, représentant au bord d'un étang vert pâle un maigre type barbu loqueteux debout dans une barque l'air de faire sa prière genre Angélus ou quelque chose comme ça misérabiliste légende LE PAUVRE PECHEUR mot à double sens comme Tu es Pierre et sur cette pierre je bâtirai mon Eglise. Colle pas en latin Petrus et petram. Peut-être en grec? Cela voulait-il dire nous misérables qui commettons des péchés ou bien nous qui ne prenons de poisson? (Simon, $1967: 310$ ).

Il s'agit, au fond, d'un condensé de trois différents passages bibliques ${ }^{9}$ parodiés. Le premier "Je suis Celui qui suis" est une déclaration divine dans le livre d'Exode ${ }^{10}$ au moment où Dieu décline son identité à Moïse sur la montagne de l'Horeb. Cette courte phrase - cinq mots -, mais très complexe est reprise in extenso par Claude Simon. Nous avons donc une ré(é)criture exacte en suivant Gignoux. En revanche, les deux autres passages dans cette parataxie sont une ré(é)criture avec variation. Ils sont dénaturés, parodiés. De fait, «il vint vers eux pauvres pêcheurs tirant leurs filets [...] mot à double sens » est une reprise d'un passage biblique de l'évangile selon Luc ${ }^{11}$. De même, «Tu es Pierre et sur cette pierre je bâtirai mon Eglise » est une métaphore christique qu'on retrouve dans l'évangile de Matthieu ${ }^{12}$.

Ajoutons à ces passages, un autre, pastiché : «[...] Moïse enveloppé dans un péplum violet jaillir du rocher frappé de son bâton l'eau d'argent et saint Louis rendant la justice laissez venir à moi les petits oiseaux ${ }^{13}[$ [...] » (Simon, $1967: 42)$.

14 Ces versets bibliques décontextualisés et juxtaposés donnent finalement dans la parodie. De cette manière, ils perdent de leur force expressive et divine ; ils deviennent simplement des textes littéraires. Ces reprises tronquées, fragmentaires et souvent falsifiées des évangiles, disséminées dans l'écriture simonienne rendent bien compte de ce que la théorie qualifie de ré(é)criture. Cette parodisation ne serait-elle pas la marque simonienne de la délégitimation des métarécits comme le prônait Jean-François Lyotard. Autrement dit, la contestation de la toute-puissance de l'église catholique romaine et son pouvoir de totalisation?

15 À côté de cette réécriture religieuse existe une très forte intertextualité dans l'écriture simonienne, en l'occurrence la ré(é)criture exacte ou avec variation des textes proustiens. Dans La Bataille de Pharsale notamment, on y retrouve des fragments de textes de Proust tels que Du temps retrouvé, Amour de Swann et Albertine disparue. Des syntagmes nominaux proustiens comme "Coiffées de hauts turbans cylindriques ", «je souffrais comme» sont récurrents dans ce texte de Simon sans variation. La 
ré(é)criture avec variation se découvre difficilement ${ }^{14}$ à travers de courts prélèvements textuels, des fragments proustiens qui sont souvent accommodés, adjoints les uns aux autres et parfois si bien intégrés au texte simonien qu'on a l'impression que ces citations d'ailleurs sont propres à l'auteur. Les portions proustiennes sont tellement bien assimilées et digérées par le texte à telle enseigne que le lecteur a du mal à identifier ces emprunts proustiens, à part la présence formelle de signes diacritiques (l'italique) qui peuvent susciter sa curiosité, à l'instar de cet extrait tiré de La Bataille de Pharsale:

Restant là à écouter le silence arrêté un instant ses yeux devant les vitrines illuminées je souffrais comme ... tissu seulement avec des pétales de poiriers en fleurs Et sur les places les divinités des fontaines publiques tenant en main un jet du glace...édicules » Rambuteau s'appelaient des pistières Sans doute dans son enfance n'avait-il pas entendu l'o et cela lui était resté. Il prononçait donc ce mot incorrectement, mais ... Entendant à de lents intervalles (toutes les dix secondes environ, les comptant $1234567 . .$. pendant un moment) l'écartement régulier de la goutte d'eau sur la grille $[. . .]^{15}$ (Simon,1969: 22) (Nous soulignons).

Ces marqueurs diacritiques s'effacent par la suite, complexifiant ainsi la ré(é)criture des textes proustiens :

grenadier qui poussait contre le mur [...] cette odeur sûre de moût d'alcool de choses en décomposition comme le premier tiers coiffées de ces hauts cylindres édicules Rambuteau s'appelaient des pistières sans doute dans son enfance n'avaitil pas entendu l'o et cela lui était que nous appelions le raidillon aux aubépines et où vous prétendez que vous êtes tombé dans votre enfance amoureux de moi alors que je vous assure Mains sous le kimono son dos comme deux colonnes dures de part et d'autre du sillon [...] (Simon,1969: 38)

On le voit, le syntagme nominal « le raidillon aux aubépines » intégré furtivement dans l'extrait précédent sera plus loin inséré dans un autre contexte: «théâtre vide de la guerre déserté silencieux pas un paysage dans les champs pas de moissonneuse dans les raidillons aux aubépines, pas de cheval blanc pas une charrette de foin » (Simon,1969: 92). Mais la ré(é)criture n'est pas seulement que textuelle; elle est aussi picturale notamment à travers les citations de peintures d'Ucello, de Breughel, de Poussin, de Piero Della Francesca et autres. Jean Rousset ajoute ainsi que «La Bataille de Pharsale se construit sur les tableaux des peintres Poussin, Ucello, Piero della Francesca, Altdorfer, Cranach, Breughel, toute une galerie de batailles peintes. »(Rousset, 1981 : 121)

En outre, il y a une ré(é)criture intratextuelle dans l'œuvre de Simon: des relations se tissent entre ses différents romans selonla triade "(r)appels - échos - réseaux ». Construit généralement à partir du précédent, le «nouveau » texte simonien fait des (r)appels ou des échos au précédent en empruntant quelques fois personnages, lieux, souvenirs, scènes, séquences, etc.La Bataille de Pharsalepar exemple fait appel à certains égards à Histoire :le dernier - selon l'ordre de parution -se construit par rapport au premier à travers les thèmes, les personnages ${ }^{16}$ (oncle Charles, Corinne, etc.).

18 Au bout du compte, la ré(é)criture, fondamentale au Nouveau roman du point de vue critique, apparaît sous bien des formes dans l'écriture simonienne. Évidente ou sournoise, élémentaire ou complexe, elle s'affirme dans ce contexte-là comme l'une des matérialités théoriques et sert, dans une certaine mesure, de voie d'accès, de rattachement de certains textes de l'écrivain à la mouvance néo-romanesque. Mais, en dépit de cette réalité théorique que nous venons d'examiner, il semble que la complexité de l'écriture simonienne ainsi que son abondance refusent qu'on cantonne et confine sa production littéraire uniquement dans l'espace néo-romanesque. Textes 
flexibles, textes ductiles, les romans de Claude Simon autorisent et appellent à de nouvelles entrées de lecture. Dans ces conditions, comme l'a dit Janet M. Paterson, il faut être attentif aux «maints aspects de [leur] production [...] Il est important d'être ouvert à [leurs] manifestations les plus hétérogènes » (Paterson, 1990 : 112).

\section{Modus operandi d'une pratique postmoderniste chez Simon}

Dällenbach mentionne à propos de Simon qu'il est un écrivain "peu néo-romanesque que possible au sens du Nouveau Roman des années soixante et de sa mythologie " (Dällenbach,1988 : 5). Autrement dit, il l'englobe dans une catégorisation plus large qui inscrit cet écrivain dans les précurseurs du postmodernisme littéraire en ne limitant pas strictement au groupe néo-roman :

Chez ces postmodernistes avant la lettre, il est symptomatique que le refus de s'en laisser accroire se traduise [en littérature qui se veut la moins idéologique et la moins «illusionniste» possible et qui délaisse le trompe-l'œil et la panoplie traditionnelle du roman «réaliste» pour reprendre elle aussi à son compte la maxime phénoménologique [...] et revendiquer surtout un Zur Sprache selbst, c'està-dire un retour à la langue même considérée d'abord dans sa logique profonde et sa matérialité productive(Dällenbach,1988:14).

Cette nouvelle approche théorique qui, dans une certaine mesure, élargit la première et qui explicite davantage les réflexions de l'écrivain trouve sa pleine satisfaction dans ses textes.

Janet M. Paterson qui a élaboré une approche conceptuelle du postmodernisme littéraire stipule que les traits distinctifs des romans postmodernes se situent au niveau de l'énonciation (narrateur, narrataire) et de l'énoncé (diégèse et code). Reconsidérons encore un texte comme Histoire. Au niveau de l'énonciation, nous avons affaire à un narrateur qui se dédouble : mêlant histoire personnelle (sa journée) factuelle à celles parentales, fictives, visualisées à partir des cartes postales, il nous entraîne dans une confusion énonciative, favorisée par la pluralité de « je ». Tantôt il apparaît comme une individualité tantôt en tant que groupe de personnages. Qui plus est, les discours directs qui surgissent çà et là dans le texte entretiennent et amplifient cette confusion. Bref, nous avons affaire à un je polyphonique comme l'attestent ces exemples : « Nous avons passé l'après-midi d'hier à la mer [...] j'ai reçu pour elle une merveilleuse broderie de Madame de Carrière Bon baisers Maman» (Simon,1967: 23) ou "Je m'embarque demain sur l'Armand-Béhec. Henri » (37) ou encore « Me voici arrivé à bon port [...] je t'embrasse Charles » (256).

21 Toutefois, dans cette polyphonie, il arrive que le je narrant soit identifiable, c'est-à-dire facilement rattachable, à un personnage grâce à une signature comme dans les exemples susmentionnés : «Henri», «Maman», etc. Dans ce jeu de camouflage, les référents du je sont donc multiples et variées ${ }^{17}$. Tout compte fait, selon Paterson, ces voix narratives finissent par s'annuler :

ces voix multiples produisent rarement un discours unifié. Elles refusent, au contraire, d'admettre une seule vision et une seule autorité et elles subvertissent toute notion de contrôle, de domination et de vérité (Paterson,1990 : 18).

Le sujet est donc fragmenté, à l'image de la construction du texte simonien comme nous le verrons. Le brouillage des voix narratives est révélateur dans la mesure où il permet de remettre en question au niveau de l'énonciation, et donc au niveau du dire, 
les notions d'autorité et de vision totalisante. En effet, le $J e^{18}$ sujet unique et incréé, expression du monothéisme, à l'origine de l'univers entier et de tout, est sabordé ici pour faire éclore une multiplicité de je qui s'affirment diversement. Cette confusion énonciative entretenue est à mettre au compte de la stratégie discursive postmoderne employée par l'écrivain qui vise à subvertir toutes les dimensions de la vérité et surtout de l'autorité/Autorité. Par ailleurs, Claude Simon semble attirer clairement l'attention du lecteur quant à la véracité de son discours. Il refuse d'imposer une ligne de lecture, une interprétation univoque de ses textes. L'écrivain s'adresse de la sorte au lecteur afin que celui-ci prenne ses distances vis-à-vis de lui. Du reste, la présence du destinataire du récit, le lecteur "encodé et virtuel » se fait sentir dans le texte, son inscription se caractérisant par l'hétérogénéité.

On note également que la surenchère de parenthèses, à l'instar de ce qui se fait dans Histoire, témoigne d'une métatextualité outrancière et a pour effet d'une part, de signaler que le narrateur est à la fois auteur et lecteur de son propre texte et de l'autre, de convoquer le lecteur. Outre ce(s) personnage(s)-lecteur(s), le texte met fréquemment en place un homologue fictif du lecteur réel: le narrataire. Selon Françoise Lepage, "[...] l'acte de communication, implicite comme dans tout discours est, souligné par des appels au narrataire " (Lepage,2003: 57) derrière lequel, selon Paterson, «se profile toujours l'ombre multiple et diffuse du lecteur réel » (Paterson, 1990 : 19). Ainsi dans ce texte, les interpellations ${ }^{19}$ servent à renforcer la fonction isomorphe du destinataire dans le récit. En fin de compte, ce texte de Claude Simon manifeste au niveau de l'énonciation une plurivocité et une hétérogénéité. Ce faisant, il s'agit d'une sorte de sollicitation au lecteur à participer à la construction de(s) sens du texte, devenant ainsi son imaginaire et son potentiel créateur. En extrapolant, on dira que les textes du Prix Nobel 1985 s'inscrivent dans la dynamique de la littérature participative tout en appelant à la posture de lecteur actif et se veulent également le miroir d'une société orientée vers la liberté, la possibilité de choix et le goût personnel. Ces éléments intrinsèques du postmodernisme littéraire apparaissent ici comme les critères fondamentaux qui doivent guider la lecture.

Considérons à présent la diégèse et le code. À ce niveau, Jacques Allard formulait que :

À ce plan de l'énoncé, de l'histoire racontée, se manifesteront donc les thèmes de l'écriture, accumulant des procédés d'autoreprésentation, passant de l'écriture d'un romancier à celle de la critique ou à l'exploitation de l'art en général (de ses figures, de sa théorie). Un autre thème incontournable est celui de la rupture, du dissentiment (Allard,1991 : 45).

La plupart des romans de Claude Simon sont auto représentatifs à l'instar de L'Acacia dans lequel se retrouvent des traces d'Histoire. Par ailleurs, Histoire s'avère l'interaction de plusieurs discours spéculaires : le récit lui-même, des passages tirés de la Bible, des messages intimes, des annonces publicitaires, des fragments de faits divers, des affiches cinématographiques, etc. Outre la spécularité, les textes simoniens apparaissent comme la somme de plusieurs genres littéraires, ce que Paterson appelle le mélange des genres :

L'aspect formel du postmodernisme littéraire représente un champ d'analyse très fertile. L'intertextualité, le mélange des genres, les mutations au niveau de renonciation (l'affirmation du «je», mais en même temps sa fragmentation), l'autoreprésentation et les jeux de langage (Paterson,1994: 81). musique... dans le même texte chez Claude Simon. Cette pluralité subvertit et favorise 
la rupture du concept d'impérialisme générique. Toujours dans Histoire, on y découvre cette multiplicité genrologique à travers des constructions parataxiques : juxtaposition d'éléments narratifs, de dialogues et surtout des fragments non littéraires selon l'expression de Paterson (1990 : 21). Dans La Bataille de Pharsale également, la parataxie prend en compte des discours publicitaires, politiques ou propagandistes ainsi que la narration elle-même :

La légende de l'affiche consiste en un seul mot imprimé en grandes lettres noires sur le fond blanc: VENCEREMOS. Cette affiche est collée plusieurs fois sur le mur d'une église. [...]. L'une d'elles répète à intervalles réguliers le mot DOLOR DOLOR DOLOR... (Simon,1969: 210).

$\mathrm{Au}$ niveau du code, des éléments comme la parodie, l'intertextualité, les structures de sur formalisation, les jeux du signifiant, le champ lexical, tout concourt à une mise en évidence de l'écriture postmoderne. La récurrence de citations étranges et étrangères ${ }^{20}$ est éloquente à ce propos.

Examinons brièvement à présent les jeux sur les signifiants, toujours à partir d'Histoire:

[...] s'étirant sur le port, disparaissant sous des charges d'ânes, et les petits ânes disparaissant sous des charges pour chevaux, et les chevaux tirant des charges pour locomotive [...] avec leurs identiques têtes de brigands, leurs identiques vêtements sombres, leur identique raideur ${ }^{21}[. .$.$] (Simon,1967 : 165).$

On remarque d'emblée l'itération de la conjonction de coordination " et " ainsi que la construction en gigogne dans laquelle la chute de la première phrase devient le début de la suivante. Dans la suite de la phrase, il y a encore une autre itération, celle du substantif «identique » qui donne l'impression d'avoir un élément répété plusieurs fois. Ces itérations semblent cadencer et rythmer les phrases dans lesquelles ils sont employés. On l'aura compris, la pratique postmoderniste se dissémine subrepticement dans les textes de l'écrivain; des premières aux dernières pages, des signifiants sont mis en valeur à de nombreux niveaux discursifs. En inventant des termes comme « pipii » dans ce contexte, n'est-ce pas une autre possibilité ouverte de subvertir l'ordre du langage et ses pouvoirs de totalisation?

Par ailleurs un autre élément significatif du renvoi à l'esthétique postmoderniste se signale : c'est le fragment. Pour Roger-Michel Allemand, il s'agit même de «[...] l'un des principaux traits de la création postmoderne» (Allemand,2013: 147). L'approche du fragment reposera sur l'espace textuel, les effets typographiques -choix des types de caractères et leur disposition, les formes des lignes sur les pages, les répartitions des noirs et blancs typographiques. En effet, la typographie ${ }^{22}$ simonienne est très expressive : les lettres capitales, les caractères en italique, les parenthèses, la mise en page (ou spatialisation) relèvent du défi et apparaissent comme des éléments fondateurs de la poétique simonienne. Ces dispositifs adoptés par l'écrivain produisent, sur le plan formel, des effets saillants que sont la rupture, l'interruption et le discontinu, souvent depuis l'incipit jusqu'à la chute du texte. En somme, la force scripturale des romans de Claude Simon repose inéluctablement sur le fragmentaire. On aboutit à des textes graphiquement différenciés, à l'exemple de Histoire, La Bataille de Pharsale, Orion aveugle ou Triptyque. La spécificité de ces textes apparaît ou se dessine à travers un appareil typographique ostensible, ostentatoire, parfois étrange et illisible comme le soutiennent les narrateurs d'Histoire (Simon,1967:118) ou d'Orion aveugle :

Aucun mot n'est lisible en entier. Il n'en subsiste que quelques fragments

énigmatiques, parfois impossibles à compléter, permettant d'autres fois une ou 
plusieurs interprétations (ou reconstitutions) comme par exemple, ABOR (LABOR, ou ABORto, ou ABORecer ?), SOCIA (SOCIAlismo, aSOCIAcíon ?) et CAN (CANdidato, CANíbal, CANker ?) (Simon,1970 : 60). contraste graphique ainsi créé entre capitales et bas-de-casse accroît la dimension visuelle du texte et tente de le libérer des carcans conventionnels. Ajoutée à ce qui précède, la ponctuation qui, sensée faciliter la compréhension du texte, s'offre dès lors comme une crise chez Claude Simon, surtout dans Histoire où le texte apparaît complètement déréglée ${ }^{3}$. Ce qui problématise davantage la grammaticalité de ce texte de Simon. Le lecteur se voit submerger ainsi par cette complexité inouïe. Quand elle existe, la ponctuation reste insolite, et obscurcit davantage la phrase ${ }^{24}$. Quant aux points de suspension, ils invitent inéluctablement le regard du lecteur par leur récurrence (soit en début de phrase soit en fin de phrase). Leur présence dans Histoire (Simon,1967 : $140 ; 134$; 147-148) ou La Bataille de Pharsale (Simon,1969 : 172-177) se lit comme une sorte de remémoration; le narrateur laissant assez de suspens(e), des sortes d'invitation, d'appels voilés au narrataire dont l'imaginaire est sollicité à travers ces marques. Exécutés en début de phrases, les points de suspension semblent traduire la vacuité de son esprit, de sa pensée et, placés à la fin, ils marquent certainement le non-dit, un effet de surprise ou un choc à venir, l'inaccompli, l'inachevé...Voyons de près cet extrait d'Histoire :

et moi : Non : des bancs. Les chaises...

... avaient sans doute été transportées (...) ou un jardin public.

[...] vous preniez (...) mâles et femelles anglo-saxons ou sud-amé...

et moi : Non : des bancs. Les chaises... (Simon,1967 : 147).

On peut le dire, les points de suspension expriment, de ce point de vue, l'état psychologique du narrateur comme l'indécision, l'hésitation, la perturbation, etc. En même temps, ils sollicitent l'imagination du lecteur. En tant que marques scriptables des trous de mémoire du narrateur, les points de suspension apparaissent aussi comme une sorte d'appels à l'imagination même du lecteur qui s'inscrit dans la posture de narrataire sur le paradigme diégétique. Par leur récurrence, cette sorte de ponctuation concourt à la subversion des codes grammaticaux et syntaxiques des textes simoniens.

S'en suit l'alinéa saillant qui est également abondant chez Simon ${ }^{25}$. Leur usage aboutit à la mise en page de chapitres entiers non marqués conventionnellement qui forment, pour ainsi dire, l'ossature des textes simoniens. Le texte final devient selon l'expression de Ludovic Janvier de "masses de $\operatorname{mots}^{26}$ ». Pour autant, ces amas de mots se communiquent entre eux, se rejettent et pour finir, forment un ensemble tout à fait cohérent. S'ajoutent également, à ce qui précède, les passages en italique. Pour l'illustration, réexaminons un extrait d'Histoire ${ }^{27}$. On le sait, l'italique n'a de contenu sémantique que dans l'acte de discours, dans l'acte de citer. Mais au-delà, son usage ici prône la sollicitation du lecteur en misant implicitement sur sa qualité interprétative. Dès lors, cette marque scriptable devient problématique en se chargeant d'une valeur polémique d'autant plus qu'il n'explicite pas une clé de lecture précise.

De même, les parenthèses jouent un rôle essentiel chez Claude Simon. Dotées d'une fonction métatextuelle, elles permettent à l'auteur de commenter son propre discours. Ces métadiscours peuvent caractériser la conscience qu'a le scripteur de ne pas être compris par son lecteur cible. Ces signes deviennent subséquemment le lieu du dédoublement de la voix de l'énonciateur, l'endroit où il décide de créer un espace graphique pour ajouter une information, une digression ou un détail par rapport au 
sens de la phrase principale ou d'une de ses parties. Toujours dans Histoire, l'itération de parenthèses entraîne le récit dans un labyrinthe tant au niveau sémantique que visuel :

(de face, fixant l'objectif du photographe [...] (ténors ou jeunes premiers), avantageux, pommadé, marmoréen et grisâtre (c'était une photo en blanc et noir dont on s'était contenté de colorer le fond après coup) surmontant le fastueux costume de poupée fait d'un morceau de soie bleu ciel [...] (fausse cursive à fioritures) [...] Niňita) (Simon,1967 : 116-117).

Les parenthèses promeuvent donc la mise en abyme du récit, confèrent une dimension labyrinthique au texte simonien et sont pour l'écrivain, un moyen de transgression de l'écriture, à l'image de la mise en page des textes, lieu également privilégié de l'innovation textuelle chez Claude Simon. Illustrons par cette mise en page extraite de Triptyque:

A partir de lui il mène ensuite deux droites aboutissant aux sommets $\mathrm{A}$ et $\mathrm{C}$ du triangle initial et il écrit au-dessous de la figure :

Pendant un moment il contemple son travail, les sourcils froncés, tournant entre ses lèvres l'extrémité du porte-plume (Simon,1973 : 27).

Cette architecture de la page blanche induit un simulacre du discontinu de la réalité, des multiples pensées, sensations, perceptions qui surgissent dans l'esprit du narrateur. Ces décrochements déclenchent une déchirure perceptible du texte et donnent à la page blanche une nouvelle architecture. Cette architecture est une tentative de reproduction du réel, créant ainsi sur la page ce que Philip Amangoua Atcha considère comme « effet de réel »:

La présentation artistique de la page donne l'impression [de voir la chose comme si c'était dans la réalité]. Cette conception de la page a une double fonction: elle permet de faire "l'économie du discours" et de créer un "effet de réel. Partant, la lisibilité gagne en profondeur et en surface (Atcha, 2012: 85).

Ce véritable travail de/sur la page blanche, cette construction très agressive, originale marque inlassablement la textualité des récits simoniens en dénotant le goût de la liberté et du jeu, le refus de ce que certains appellent «beau style » et en développant une hétérogénéité architecturale du texte.

On l'aura compris, Claude Simon s'est fait fort d'expliquer le processus de création de ses textes lorsque l'occasion lui en a été donnée. La préface d'Orion aveugle, qu'il a écrit de lui-même, constitue une preuve parmi tant d'autres. Il ressort de toutes ses explications que l'auteur n'a jamais été un fervent adepte de la théorisation, se contentant uniquement de rester dans sa posture d'écrivain artiste. S'il reconnait certaines influences comme celle d'Alain Robbe-Grillet sur sa production littéraire, Simon n'a eu de cesse de se démarquer, de se singulariser à travers des textes dont l'aspect visible, formel et l'«histoire » donnée à lire sont frappés par le sceau du renouvellement dû à " une insatisfaction vis-à-vis de la forme traditionnelle du roman et par la recherche, d'abord infructueuse, d'une alternative qui lui convienne" (Duncan, 2006 : XVI).

Une telle production littéraire ne peut donc rester indifférente vis-à-vis de la critique. Alastair B. Duncan reconnaît que dans les années 1970, la critique proche du Nouveau roman a su éclairer des aspects de la forme des romans de Simon. Ayant montré ses limites, une nouvelle critique verra le jour avec Lucien Dällenbach et autres afin d'élargir et d'approfondir dans toutes ses dimensions, la compréhension de l'œuvre 
simonienne. Ainsi, en tout état de cause, l'écriture simonienne prend en compte aussi bien l'esthétique du Nouveau roman prônée par Ricardou et Robbe-Grillet que celle du postmodernisme littéraire dont parle Dällenbach. L'œuvre du prix Nobel de 1985 possède in fine ces deux labels : ses textes ont effectivement les «pieds » dans l'univers néo-romanesque, mais la "tête" dans le postmodernisme littéraire. Les textes de Simon sont ductiles, ils résistent à toute tentative d'enfermement et de totalisation. Ouverts et flexibles, ils sont très favorables à de nouvelles entrées de lecture; une lecture non soumise au principe de l'unicité du sens dans une unité signifiante, mais propice à la pluralité irréductible de sens. C'est pourquoi nous convenons avec LucieMarie Magnan et Christian Morin qui soutiennent que «[...] le Nouveau Roman constitue une espèce de transition entre le modernisme et le postmodernisme, si bien que l'œuvre de certains Nouveaux romanciers participe tantôt à l'univers du modernisme, tantôt à celui du postmodernisme » (Magnan et Morin, 1997 : 19).

\section{BIBLIOGRAPHIE}

ALLARD, Jacques, « Postmodernités », Lettres québécoises : la revue de l'actualité littéraire, 62, 1991, p. 44-45. « Disponible sur » (http://id.erudit.org/iderudit/38440ac. ), (consulté le 13/12/19). ALLEMAND, Roger-Michel, « Nouveau roman, Nouveau Monde », Laboratoire Babel, Université du Sud-Toulon-Var, 8, 3, 2013. « Disponible sur », (www.revue-analyses.org,.), (consulté le 12/12/19). ATCHA, Amangoua Philip, «L'intermédialité littéraire dans La mémoire amputée de Were were Liking », « in » ROBERT FOTSING MANGOUA (éd.) Écritures camerounaises francophones et intermédialité, Yaoundé, Ifrikiya, 2012.

CHAUTARD, Paul, "Orion aveugle marchant vers la lumière du soleil levant » : une théopoétique des romans de Claude Simon, HEYER RENÉ (dir.), Thèse de doctorat, Université de Strasbourg, 2012.

DÄLlENBACH, Lucien, Claude Simon, Paris, Seuil, 1988.

FONTVIEILLE, Agnès, « Les reliefs typographiques dans Nadja, sur quelques emplois de l'italique », « dans », L’histoire et la géographie dans le récit poétique, Paris, Presses Univ Blaise Pascal, 1997, p. 257-267. « Disponible sur », (http://books.google.ci/books.), (consulté le 15/12/19).

GIGNOUX, Anne-Claire, La récriture : formes, enjeux, valeurs autour du Nouveau Roman, Paris, Presses Universitaires de Paris-Sorbonne, 2003.

GoSSELIN, Katerine, «Claude Simon et le roman « nouveau », « in » DIRKX PAUL, MOUGIN PASCAL (dirs), Claude Simon : Situations, Lyon, ENS Éditions, 2011, p. 69-85.

JANVIER, Ludovic, « Le temps d'une histoire », La Quinzaine littéraire, 25, 1er avril 1967.

LEPAGE, Françoise, La littérature pour la jeunesse, 1970-2000, Montréal, Fides, 2003.

MAGNAN Lucie-Marie, MORIN Christian, Lectures du postmodernisme dans le roman québécois, Québec, Nuit blanche Éditeur, 1997. 
N'DA, Pierre, Méthodologie et guide pratique du mémoire de recherche et de la thèse de doctorat, Paris,

L'Harmattan, 2012.

PATERSON, Janet M., « Le Postmodernisme québécois. Tendances actuelles », Études littéraires, 27, 1, 1994, p. 77-88. « Disponible sur », (http://id.erudit.org/iderudit/501069ar.), (consulté le 13/12/19).

PATERSON, Janet M., Moments postmodernes dans le roman québécois, Ottawa, Les Presses de l'Université d'Ottawa, 1990.

ROBBE-GRILLET, Alain, Pour un nouveau roman, Paris, Minuit, 1961.

ROUSSET, Jean, Passages. Échanges et transpositions, Paris, Corti, 1990, p. 165-175.

ROUSSET, Jean, « Histoire de Claude Simon, Le jeu des cartes postales ", Revue suisse des littératures romanes, 1981, p. 121-134. « Disponible sur »,(http://dx.doi.org/10.5169/seals-248350), (consulté le $01 / 12 / 19)$.

SELLIER, Phillipe, "Qu'est-ce qu'un mythe littéraire ? ", " dans », Littérature, La farcissure.

Intertextualités au XVIe siècle, 55, 1984, p. 112-126. « Disponible sur », (http://www.persee.fr/doc/ litt_0047-4800_1984_num_55_3_2239), (consulté le 01/12/19).

SIMON Claude, ALPHANT, Marianne, « La route du Nobel », Libération, 10 décembre 1985.

SIMON Claude, JOGUET Monique, « Entretiens avec Claude Simon », L'en-je lacanien, 2007, n 8, p. 165-196.

SIMON, Claude, « « Claude Simon, à la question », réponses aux questions des participants au Colloque de Cerisy (1974)», « dans », Lire Claude Simon, Paris, U.G.E.,10/18, 1975, p. 403-431.

SIMON, Claude, Histoire, Paris, Minuit, 1967.

SIMON, Claude, L'Acacia, Paris, Minuit, 1989.

SIMON, Claude, La Bataille de Pharsale, Paris, Minuit, 1969.

SIMON, Claude, Orion aveugle, Genève, Skira, 1970.

SIMON, Claude, Triptyque, Paris, Minuit, 1973.

SIMON, Claude, CEuvres, Paris, Gallimard, 2006.

YOCARIS, Ilias, « L'impossible totalité. Une étude de la complexité dans l'œuvre de Claude Simon », Loxias,8, 2005, p. 1-10. « Disponible sur », (http://revel.unice.fr/loxias/index.html?id=107), (consulté le 28/12/19)

\section{NOTES}

1. ${ }^{1}$ Il s'agit du premier volume/tome établi par Alastair B. Duncan, avec la collaboration de Jean H. Duffy paru aux éditions Gallimard en 2006.

2. ${ }^{2}$ Nous entendons par «transition »la « manière de passer progressivement d'un ton à un autre, de l'ombre à la lumière » ou le " passage d'un plan à un autre ».

3. ${ }^{3}$ Il s'agira de: Histoire (1967), La Bataille de Pharsale (1969), Orion aveugle (1970) et Triptyque (1973), L'Acacia (1989).

4. ${ }^{4}$ Il s'agit de Robert Pinget, Michel Butor, Nathalie Sarraute.

5. ${ }^{5}$ Entretiens réalisés en 1976 par Monique Joguet pour France Culture, retranscrits par Brigitte Hatat, psychanalyste à Reims, à partir de leur enregistrement audio, rediffusés dans le cadre de 
l'émission Mémorables, du 13 au 15 juillet 2005, suite à la disparition de Claude Simon survenue le 5 juillet 2005.

6. ${ }^{6}$ Selon Roger-Michel Allemand, l'article écrit par Émile Henriot intitulé le «nouveau roman » est un « éreintement et utilise l'expression de façon dépréciative " (Voir Roger-Michel Allemand «Nouveau roman, Nouveau Monde», Laboratoire Babel, Université du Sud-ToulonVar,www.revue-analyses.org,vol. 8, no 3, automne 2013, p. 143).

7. ${ }^{7}$ Beaucoup de travaux de recherche (articles, mémoires, thèses) ont apporté la preuve du rapprochement des textes de Claude Simon et le Nouveau roman entre autres celui de Jean Ricardou, Lire Claude Simon : Colloque de Cerisy-la-Salle, Paris, Les Impressions nouvelles, 1986.

8. ${ }^{8}$ Nous emploierons dans notre analyse le terme de ré(é)criture pour désigner ce que AnneClaire Gignoux qualifie de récriture.

9. ${ }^{9}$ La version de la Bible consultée est la version Louis Segond.

10. ${ }^{10}$ Voir exactement le livre d'Exode chapitre 3 le verset 14.

11. ${ }^{11}$ Voir exactement le livre de Luc chapitre 5 les versets 2 à 11 . Ce même passage peut être retrouvé dans les évangiles de Matthieu (Matthieu 4 versets 18 à 22), Marc (Marc 1 versets 16 à 20) et Jean (Jean 1 versets 35 à 43).

12. ${ }^{12}$ Voir le livre de Matthieu, le chapitre 16 le verset 18.

13. ${ }^{13}$ Il y a d'autres parodisations de passages bibliques aux pages $45 ; 39 ; 147 ; 310 ; 395$. Ce passage apparaît comme un condensé de la vie de Moïse.

14. ${ }^{14} \mathrm{Il}$ faut que le lecteur soit un lecteur averti de Claude Simon.

15. ${ }^{15}$ La partie en gras ici est en réalité en italique dans le texte original.

16. ${ }^{16}$ Nous avons une réécriture avec variation du portrait de la femme de Van Velden. Dans Histoire, on peut lire à la page 28 : «La russe-ou la Hongroise, ou la polonaise [...]la femme plate, avec ses souliers plats, son flasque tricot

17. ${ }^{17}$ Il peut s'agir du narrateur lui-même, de ses parents, des connaissances de ses parents.

18. ${ }^{18}$ Voir les passages bibliques cités plus haut.

19. 19 Voir Histoire respectivement aux pages 40, 51,52, 87, 93. Quelques exemples d'interpellations : «ses yeux comment s'appelle ce phénomène qui empêche les liquides de... »; «Devinez »; « (quel est ce type qui disait que tout ce qui est important dans la vie se fait à l'aide de) »; « Mais exactement, exactement? » « Mais exactement?»

20. ${ }^{20}$ Le plus souvent, les citations sont en latin, en grec, en espagnol, en français, en russe.

21. ${ }^{21}$ Un passage de la même construction ludique se trouve à la page $263:$ «[...] en pain d'épice du château médiéval en pain d'épice des maisons en pain d'épice ou ocre ou roses ou blanches ou brique de l'eau [...]».

22. ${ }^{22}$ Dans notre analyse, il sera plus question de la mise en forme de l'écrit. Même si la typographie conditionne l'accès au texte tout en mettant à nue la problématique de la lisibilité, c'est, dans le cas de Claude Simon, son aspect original qui attire l'attention d'une part, du narrateur simonien et de l'autre, du lecteur et surtout sa dimension esthétique sur laquelle nous insisterons.

23. ${ }^{23}$ Le dérèglement réside dans l'absence ou l'inadéquation de la ponctuation.

24. ${ }^{24}$ Les citations mentionnées à cet effet sont respectivement aux pages 14, 78, 87, 282, 390.

25. ${ }^{25} \mathrm{Il}$ faudra certainement nuancer et relativiser dans le cas de Triptyque.

26. ${ }^{26}$ Ludovic Janvier, La Quinzaine littéraire, 1er avril 1967.

27. ${ }^{27}$ Voici ce passage : «[...] par rapport à l'axe du placard-annonce riant aux éclats la bouche grande ouverte un si violent coup de glaive que la pointe en ressortit par la nuque Je croyais me

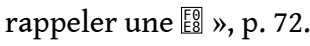




\section{RÉSUMÉS}

La réception de l'œuvre littéraire de Claude Simon a eu deux tendances :la tendance « Ricardou » inscrit strictement l'œuvre dans le Nouveau roman. La tendance «Dällenbach», dans une perspective plus large, considère l'œuvre comme avant-gardiste du postmodernisme littéraire. Cet article examine ces deux tendances pour aboutir à cette finalité : la transcendance, la ductilité de la création simonienne.

The reception of Claude Simon's literary work has had two tendencies: the Ricardou tendency strictly inscribes the work in the New Novel (French Nouveau roman). The Dällenbach trend, in a broader perspective, sees the work as avant-garde of literary postmodernism. This article examines these two tendencies to achieve this finality: the transcendence, the ductility of the Simonian creation.

INDEX

Mots-clés : réception, nouveau roman, postmodernisme, ré(é)criture, stratégies postmodernes Keywords : reception, new novel, postmodernism, re-writing, postmodern strategies

\section{AUTEUR}

\section{SINAN ANZOUMANA}

Université Félix Houphouët-Boigny, Côte d'Ivoire 